\title{
Investigation of Attenuation and Directional Characteristics of Millimeter Wave Propagation in Urban Environment Using Ray Tracing Technique
}

\section{Segni Merga Adula}

Haramaya University

Feyisa Debo Diba

Adama Science and Technology University

Atli Lemma Gebretsadik ( $\nabla$ atli.lemma@outlook.com )

Haramaya University https://orcid.org/0000-0001-9436-1242

\section{Research Article}

Keywords: High densification, Mobile speed estimation, Envelop peaks and troughs, Resource management, Handoff management

Posted Date: July 1st, 2021

DOl: https://doi.org/10.21203/rs.3.rs-380243/v1

License: (c) (i) This work is licensed under a Creative Commons Attribution 4.0 International License. Read Full License 


\section{Abstract}

Future high densification wireless networks come with high handoff rates, which require knowledge of mobile speed. Mobile speed estimation is crucial for optimizing handover to reduce call drops and network signaling flow, optimize traffic scheduling, improve quality of service, achieve resource optimization, mobility load balancing, channel quality feedback enhancement, and energy efficiency. In this paper, we present a low complexity mobile speed estimation model using count of peaks and troughs of the received signal envelop. We simulated the model in Matlab ${ }^{\circledR}$ and our result shows that the model has a maximum error of $0.25 \mathrm{~m} / \mathrm{sec}$. The model has two advantages. First, it does not require measurement of the received signal power; it only counts envelop peaks and troughs. Second, the model is independent of dc offset inherent in the radio receivers. However, the model has one limitation- it does not give the crossing component of a mobile's velocity.

\section{Introduction}

The study of mm-wave propagation, i.e. both attenuation and directional characteristics, are important for $5 G$ and beyond wireless communication system design and network deployment [1] [2] [3]. Besides, sitespecific propagation studies are useful to ascertain optimum coverage of network base stations [1]. In addition, scenario-specific study of mm-wave propagation loss and directional spread is desired to provide reliable services in, for example, high speed trains [2]. Also, to overcome the high attenuation at $\mathrm{mm}$-wave frequencies, directional antennas and beamforming are used and thus we require to know the directional spread characteristics of the wave at these high frequencies [3] .

In $5 \mathrm{G}$ and beyond wireless communication network deployment and optimization, there is a problem of accurately estimating the field distribution and directional spread of $\mathrm{mm}$-wave propagation [1]. Millimeter wave signals are highly attenuated by building materials and vegetation and thus the propagation range is relatively small. That means millimeter wave signals experience high path loss when propagated through long distance and complex environments. Also, the reflection characteristics of building materials depend on polarization, electrical properties of materials, antenna beam width, building structures and location of transmitter and receiver [4]. Thus, the attenuation of mm-waves in outdoor scenarios due to buildings and vegetation are more severe than for classical microwave frequencies [1]. Radio waves propagate in different paths with different delays. Hence, these multipath signals do not align with the line-of-sight signal and the combined signal will be smeared in time causing delay spread. Delay spread is the second moment of multipath power delay profile and causes inter symbol interference (ISI) [5]. Consequently, the spread in directivity of mm-wave signals with increased coverage requires us to study the directional spread characteristics to determine the spacing between base stations for collaborative operation, i.e. the spatio-temporal distribution of the waves is a key planning factor. This will help us in spatial scanning of mobiles by massive and smart base-station antennae [3]. Importantly, to adequately assess the performance of $5 \mathrm{G}$ and beyond wireless systems and networks, there is a need to develop mm-wave path loss and directional spread models [6]. 
Ray tracing method is based on geometrical optics, geometric theory of diffraction, and uniform theory of diffraction used to predict the propagation properties of radio wave [7] [8]. An-Yao Hsiao in[1] presents an urban outdoor ray tracing simulations using global ray tube approach rather than using individual rays and hence the method has better computational efficiency. In addition, in this work, field measurements were made and these data were used to calibrate the ray tracing simulation. Here, although similar results were obtained in both approaches, fast fading behaviors are different. However, the work did not address the directional spread characteristics of mm-wave signals, which affects the performance of $5 \mathrm{G}$ and beyond networks. The work in[3] empirically investigated the directional multipath propagation characteristics of mm-waves for a typical urban scenario. Specially, it determined delay and angular spread. Further, microcellular radio channel characterization is measured at $60 \mathrm{GHz}$ as presented in[9] using directional steering antennas at the mobile end. In this work, path loss and delay spread strongly depend on the antenna pattern. Nevertheless, the approach is expensive and time consuming especially it becomes prohibitively expensive for closely spaced $5 \mathrm{G}$ and beyond base stations. Finally, the work in[6] presented a combination of empirical measurement and ray tracing methods to predict path loss at various mm-wave frequencies. But, it did not deal with the directional characteristics of mm-waves as function of distance between transmitters and receivers. In [10], studies of outdoor propagation with different scenarios at $60 \mathrm{GHz}$ frequency bands is carried out. This work considered scattering and diffraction as the main propagation phenomenon causing sever multipath in an urban scenario. The work deduced that, in urban environments, path loss and delay spread can be reduced substantially and are a function of antenna directivity and transmitter-receiver separation. The propagation characteristics of outdoor wireless channels at 28, 39, 60 and $73 \mathrm{GHz}$ are studied for Line of Sight (LOS) and Non Line of Sight (NLOS) in [11]. The authors in this work concluded that LOS has a high receiving capacity and lower path losses than NLOS. Also, they found out that high frequencies are affected more than low frequencies and that meant increase in the path loss and decrease in the received power.

Currently, ray tracing is preferred for propagation modeling more than other methods such as the empirical models. It can be applied to many of the propagation scenarios in wireless communications and play an important role in the future propagation modeling tools. This is because it is useful for tackling complicated propagation environments at high frequencies [7]. In our paper, the economic approach of predicating attenuation (path loss) and directional spread are investigated using an advanced ray tracing technique. The technique can realistically and economically be done for all basestations of a typically $5 \mathrm{G}$ and beyond network. The rest of the paper is organized as follows. Methodology is described in Sect. 2. The simulation result on path loss, delay spread, and power delay profile parameters are analyzed and discussed in Sect. 3. Finally, Sect. 4 summarizes our findings.

\section{Methodology}

In this work, we modeled the biggest outdoor market place in Addis Ababa, Ethiopia, using the propagation software known as WinProp. The input to the software is the space map of the market. In the model, buildings are represented with default electrical properties that correspond to standard concrete, which is the main component of most commercial buildings. The input map consists of 561 building 
blocks. In our work, the default material properties for all buildings are utilized. The material properties of the buildings fed to the software are the electrical properties of concrete such as dielectric permittivity and electrical conductivity. Then, building database is pre-processed using the package called WallMan (built into WinProp) to divide building walls into tiles and the edges into horizontal and vertical segments. During the database pre-processing, there were some settings that affected the prediction accuracy and computation time. The settings made in the software include multiple interactions between objects to ensure the visibility relations; the latter is extended over to more than one single interaction (reflection or diffraction) between transmitter and receiver. Furthermore, processing of the database includes the selection of different types of propagation models, which reduce the computation time during the prediction of propagation characteristics. In this work, we have chosen the three-dimension intelligent ray tracing (3D IRT) model built into WallMan. In our work, we have not modelled the effect of foliage, cars, and human beings. However, their effect on actual wireless communications is well understood. Also, ground reflection is not considered.

\section{Results And Discussions}

The simulation parameters of our model are described in Table 1. The table shows the main parameters that can be set in WinProp. After pre-processing of the digital input map using WallMan, it was loaded into the package ProMan (still another part of WinProp). Then, delay spread and path loss (attenuation) are simulated and analyzed on the basis of the locations of transmitter assigned in ProMan. In this work, the received power, path loss, delay spread and power delay profile of the urban scenario mentioned above are estimated.

\section{Table 1}

Parameters for simulation

In our simulation, millimeter wave base stations are placed at different heights in the simulation scenario, i.e. Merkato, Addis Ababa, within WinProp suite. In particular, the transmitting antenna heights are $20 \mathrm{~m}$, $36 \mathrm{~m}$ and $31 \mathrm{~m}$ for site 1, site 2 and site 3 respectively as shown in Fig. 3. The IRT (Intelligent Ray Tracing) simulation is done with standard values of permittivity and conductivity as indicated in Table 1. Furthermore, Fig. 2 shows the distribution of receiver RX1-RX12.

Figure 1. Transmitter location and receivers distribution

\subsection{Received Power}

Receiver points RX2, RX5, RX8 and RX12 are in line-of-sight (LOS) positions and other receivers are in non-line-of-sight (NLOS) positions. Then, the data shown in the Fig. 2 was obtained and shows the effect of changing distance and frequency. Increasing the distance and frequency from transmitting antenna leads to decrease in received power. In addition, the power degradation in the NLOS regions resulted from the reflections by the buildings. 
Figure 2. Predicted received power at millimeter frequency bands

Then, we have noticed that the number of rays received by all receivers obviously decreases when there are buildings in between the transmitter and receivers. This is because, buildings highly attenuate the signal and thus receivers in NLOS get minimum power. In NLOS, fewer rays are received and hence the received power is lower. Furthermore, as shown in Fig. 3, RX2, RX5, RX8 and RX12 receive several rays, where most of them are due to reflections. Thus, the mean power received at those receivers are good.

Figure 3. Number of rays received per receiver's locations

\subsection{Path Loss}

Figure 4 shows the relation between path loss and distance for LOS case at different millimeter frequencies. It can be observed that the predicted path loss increases as TX1-RX2 distance increases. In addition, path loss increases with increase in distance and frequency.

Figure 4.Path loss from TX1 site 1 to RX2 at different frequency

\subsection{Delay Spread}

Millimeter waves reach the receiver through different propagation ray paths and each path experiences different time of travel. When delay spread is too large, it causes inter-symbol interference (ISI) which limits data rate. In this work, we observed that the delay spread decreases as the distance between the transmitter and the receiver increases. In fact, we know that delay spread will mostly be due to reflections. As shown in Fig. 5, delay spread decreases with increasing distance and frequency at different millimeter frequency bands. In LOS scenario, the delay spread decreases with increasing distance. But, the rate is different from that of NLOS because the signal passes through less number of barriers.

Figure 5. Delay Spread versus distance at different frequency

\subsection{Power Delay Profile}

The received power with different delay along the TX1-RX2 separation distance is shown in Fig. 6 . The maximum excess delay is less than $640.96 \mathrm{~ns}$ for all received paths at RX2 with received power less than $-106.7 \mathrm{dBm}$. When high gain directional antenna is used, most power falls in the LOS path. The receiver RX2 appears in the LOS path and represents the first path in the LOS scenario for the distance less than $300 \mathrm{~m}$. This receiver is located in the NLOS path with a distance more than $300 \mathrm{~m}$ and the received power is less than $-125 \mathrm{dBm}$. Here, many paths fall in excess delay of more than $800 \mathrm{~ns}$.

Figure 6. Power delay profile at Tx1-Rx2 separation

\section{Conclusion}

Close investigation of millimeter wave outdoor propagation is important for the design and planning of future high densification, high data rate, and low latency heterogeneous wireless communication 
networks. Among others, accurate results are required on path loss, delay spread, received power, and power delay profile for optimum placement of closely spaced base station for future networks. In this work, the intelligent ray tracing (IRT) method is used to investigate the attenuation and directional characteristics of millimeter waves in an urban environment. We have simulated the propagation in WinProp (with built in WallMan and ProMan) and our results are in agreement with earlier efforts elsewhere. We have also found out that the propagation is mainly due to direct path as well as single and two-times reflections. In summary, based on our simulation results, we have observed that $66 \mathrm{GHz}$ produce the best outcome for delay spread, path loss and received power when compared with other frequencies. Increasing the frequency will increase the delay spread and path loss. Also, strength of received power decreases with increase in frequency and propagation paths.

\section{Declarations}

\subsection{Funding}

There is no grant funding received for this reseach. However, the first author received a small amount of money from the School of Graduate Studies of Haramaya University as a support to the execution of the masters thesis of the first author.

\subsection{Conflict of Interest}

We declare that there is no conflict or competing interests related to the conduct, methodology, and results of this research

\subsection{Availability of Data and Materials}

The data generated and associated materials in this research can be availed when requested.

\subsection{Code Avaialbility}

We have used the software known as WinProp with built in WallMan and ProMan. We have not written any custom code. However, we have configured and used the software to meet our needs.

\section{ACKNOWLEDGMENT}

We would like to thank Haramaya University for providing funding for this research. Specially, we would like to thank the School of Graduate Studies of the University.

\section{References}

1. Hsiao, A. Y., Yang, C. F., Wang, T. S., Lin, I., \& Liao, W. J. (2017), Ray Tracing Simulations for Millimeter-wave Propagation in 5G Wireless Communications, 2017 IEEE International Symposium on Antennas and Propagation \& USNC/URSI National Radio Science Meeting (pp. 1901-1902). 
2. Nakamura, K., Iwasawa, N., Kawasaki, K., Shibagaki, N., Sato, Y., \& Kashima, K. (2017), Study of the New Application Using the Millimeter-wave in the Railway, 2017 IEEE Conference on Antenna Measurements \& Applications (CAMA), (pp. 20-23).

3. Kim, M. D., Liang, J., Lee, J., Park, J., \& Park, B. (2016), Directional Multipath Propagation Characteristics Based on $28 \mathrm{GHz}$ Outdoor Channel Measurements, 2016 10th European Conference on Antennas and Propagation (EuCAP), (pp. 1-5).

4. Lee, J., Kim, K. W., Kim, M. D., Park, J. J., Yoon, Y. K., \& Chong, Y. J. (2019). Measurement-based Millimeter-wave Angular and Delay Dispersion Characteristics of Outdoor-to-Indoor Propagation for 5G millimeter-wave Systems. IEEE access : practical innovations, open solutions, 7, 150492-150504.

5. Yang, Y., Xu, J., Shi, G., \& Wang, C. X. (2018), 5 G Wireless Systems, Springer, USA.

6. Thomas, T. A., Rybakowski, M., Sun, S., Rappaport, T. S., Nguyen, H., Kovacs, I. Z., \& Rodriguez, I. (2016), A Prediction Study of Pathloss Models from 2-73.5 GHz in an Urban-macro Environment, 2016 IEEE 83rd Vehicular Technology Conference (VTC Spring), (pp. 1-5).

7. Yin, X., \& Cheng, X. (2016), Propagation Channel Characterization, Parameter Estimation, and Modeling for Wireless Communications, IEEE-John Wiley \& Sons.

8. Fathy, A., Yahya, A., \& Ragai, H. (2014), Deterministic UWB Channel Modeling Using Ray Tracing Approach, 2014 International Conference on Engineering and Technology (ICET) (pp. 1-4).

9. Cid, E. L., Taboas, M. P., Sanchez, M. G., \& Alejos, A. V. (2016). Microcellular Radio Channel Characterization at $60 \mathrm{GHz}$ for $5 \mathrm{G}$ Communications. IEEE Antennas and Wireless Propagation Letters, 16, 1476-1479.

10. Singh, H., Prasad, R., \& Bonev, B. (2018). The Studies of Millimeter-waves at $60 \mathrm{GHz}$ in Outdoor Environments for IMT applications: A State of Art. Wireless Personal Communications, 100(2), 463474.

11. Muttair, K. S., Al-Ani, O. A. S., \& Mosleh, M. F. (2019), Outdoor Millimeter-wave Propagation Simulation Model for $5 \mathrm{G}$ Band Frequencies, 2019 2nd International Conference on Electrical, Communication, Computer, Power and Control Engineering (ICECCPCE), (pp. 40-45).

\section{Tables}

Table 1: Parameters for simulation 


\begin{tabular}{|l|c|}
\hline \multicolumn{1}{|c|}{ Parameters } & Values \\
\hline Relative Permittivity & 4 \\
\hline Relative Permeability() & 1 \\
\hline Conductivity() & $0.01 \mathrm{~S} / \mathrm{m}$ \\
\hline Minimal diffraction loss of incident rays & $8 \mathrm{~dB}$ \\
\hline Reflection loss & $9 \mathrm{~dB}$ \\
\hline Maximum diffraction loss of incident rays & $15 \mathrm{~dB}$ \\
\hline Diffraction loss of diffracted rays & $5 \mathrm{~dB}$ \\
\hline Transmission loss (Walls) & $20 \mathrm{~dB}$ \\
\hline Factor for determination of breakpoint distance & $4 \Pi$ \\
\hline Operating Frequency & $66-73 \mathrm{GHz}$ \\
\hline Transmitting Power & $30 \mathrm{~dB}$ \\
\hline Antenna Gain & $16.5 \mathrm{~dB}$ \\
\hline
\end{tabular}

\section{Figures}

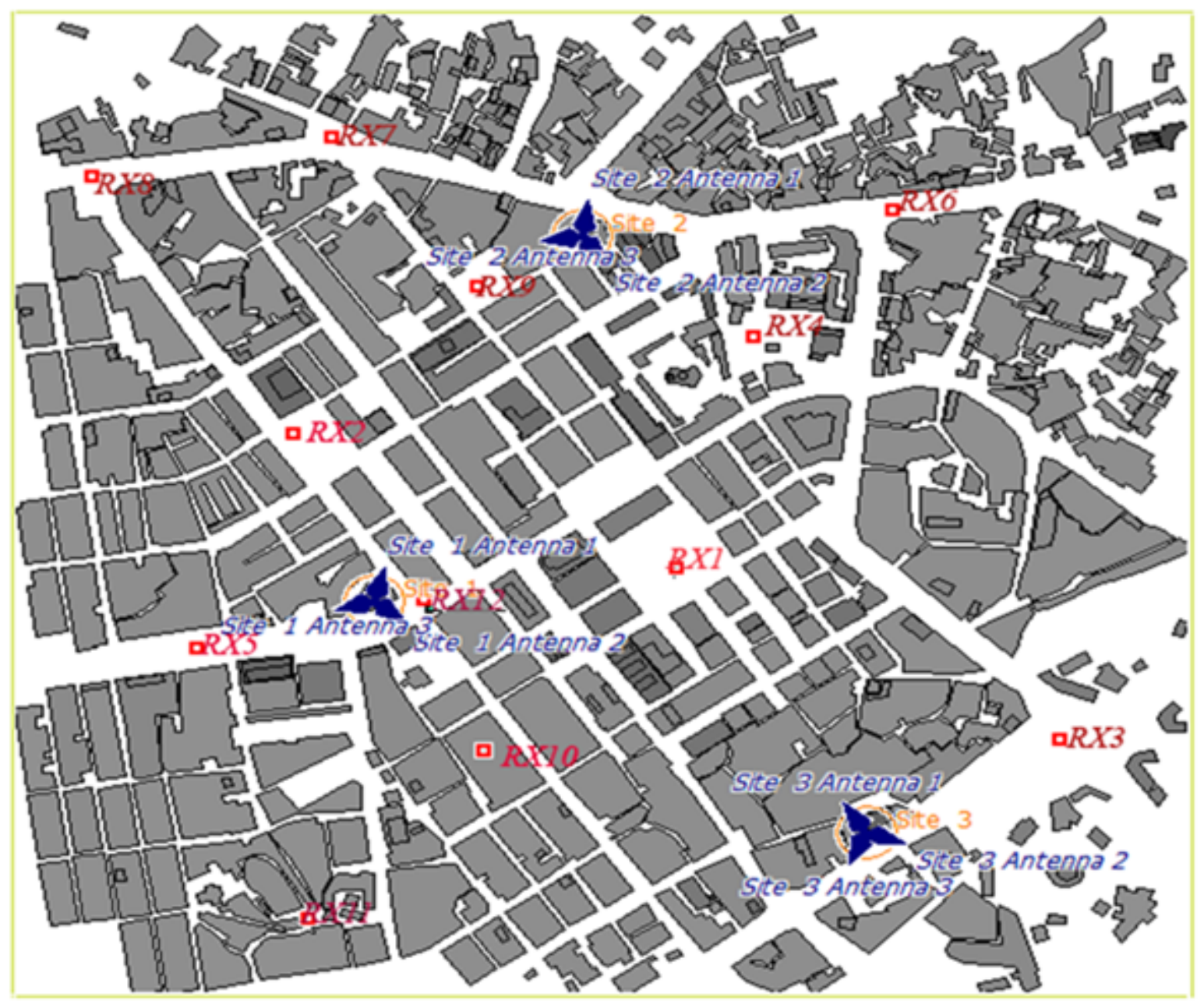


Figure 1

Transmitter location and receivers distribution

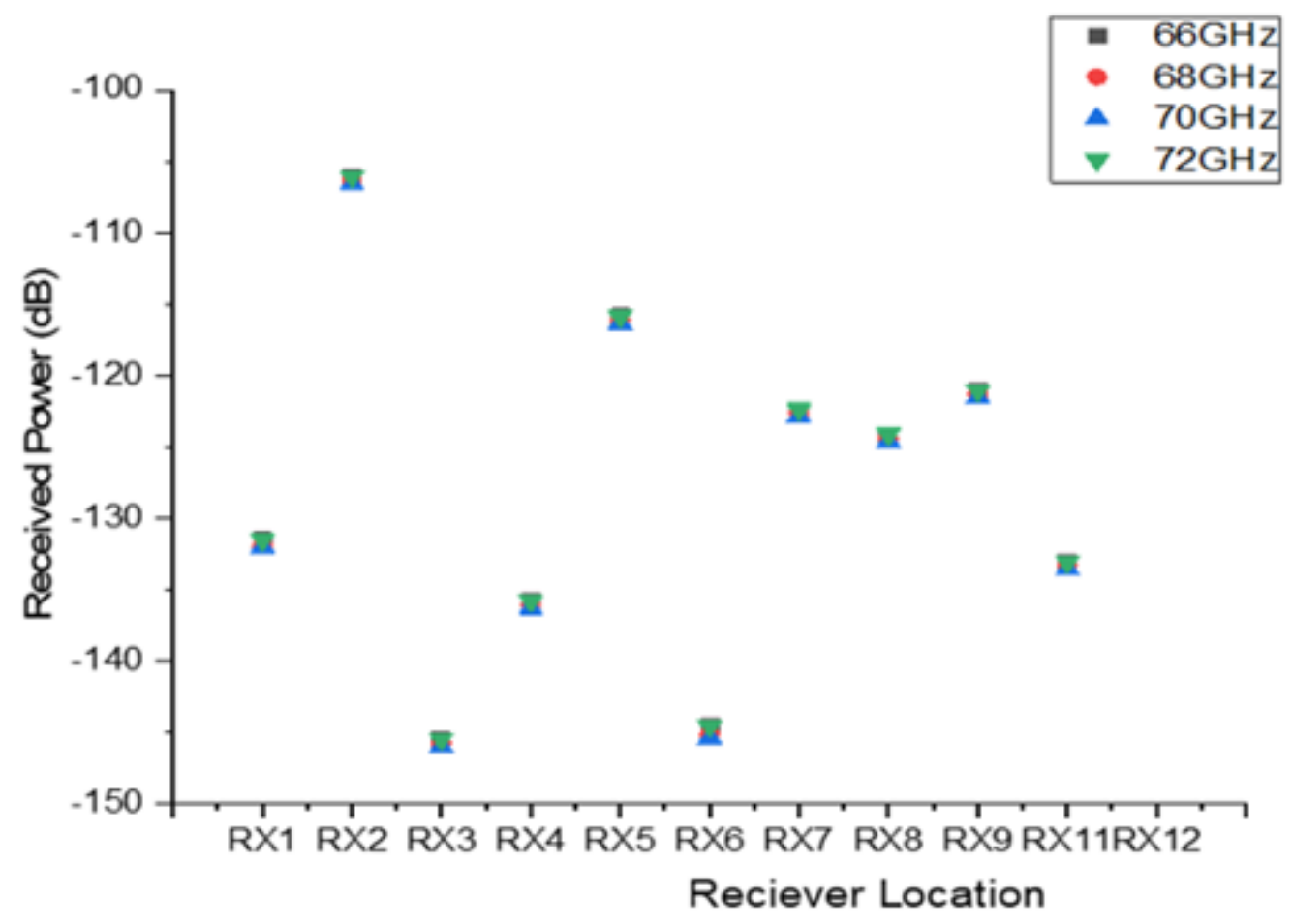

Figure 2

Predicted received power at millimeter frequency bands 


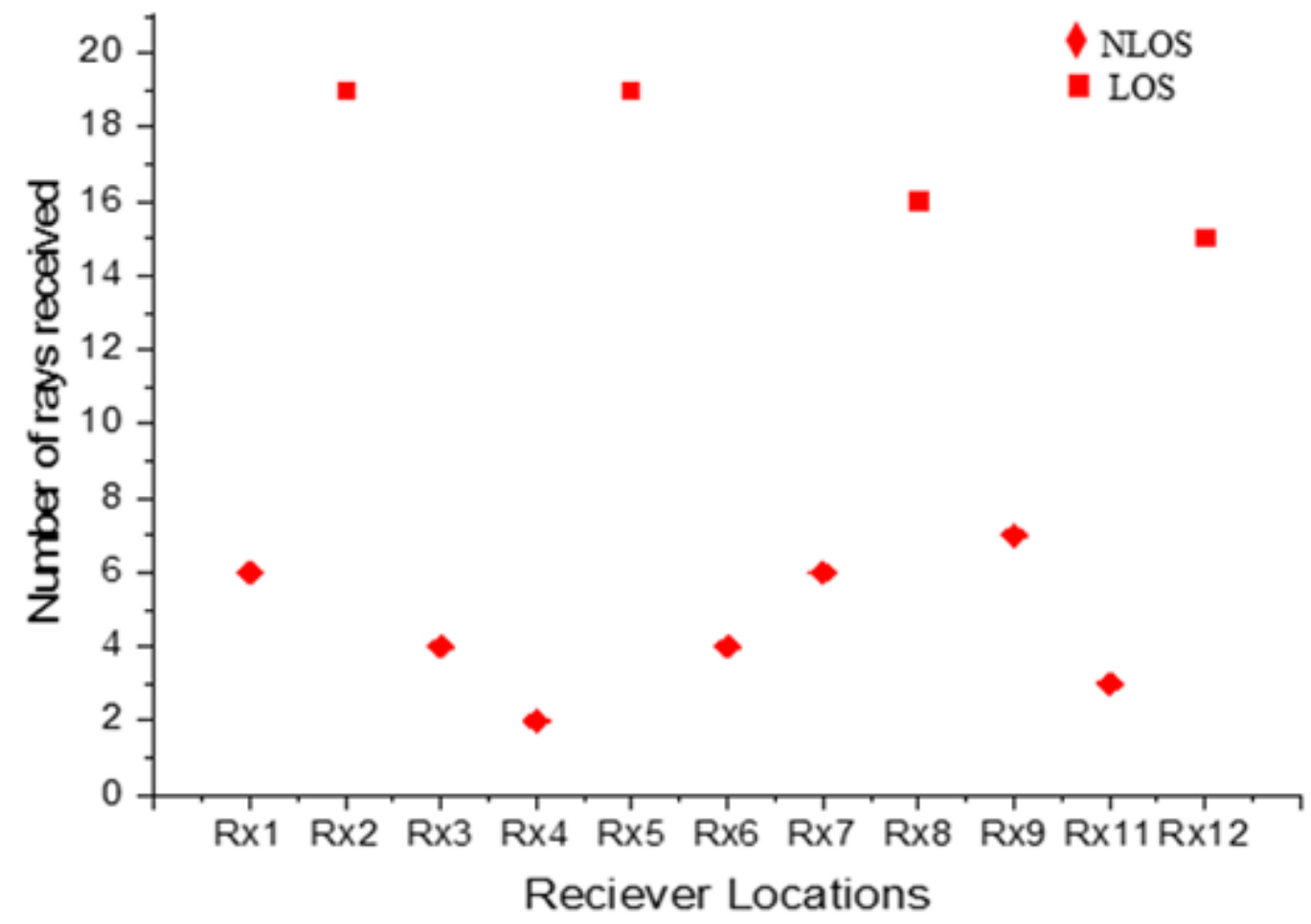

Figure 3

Number of rays received per receiver's locations 


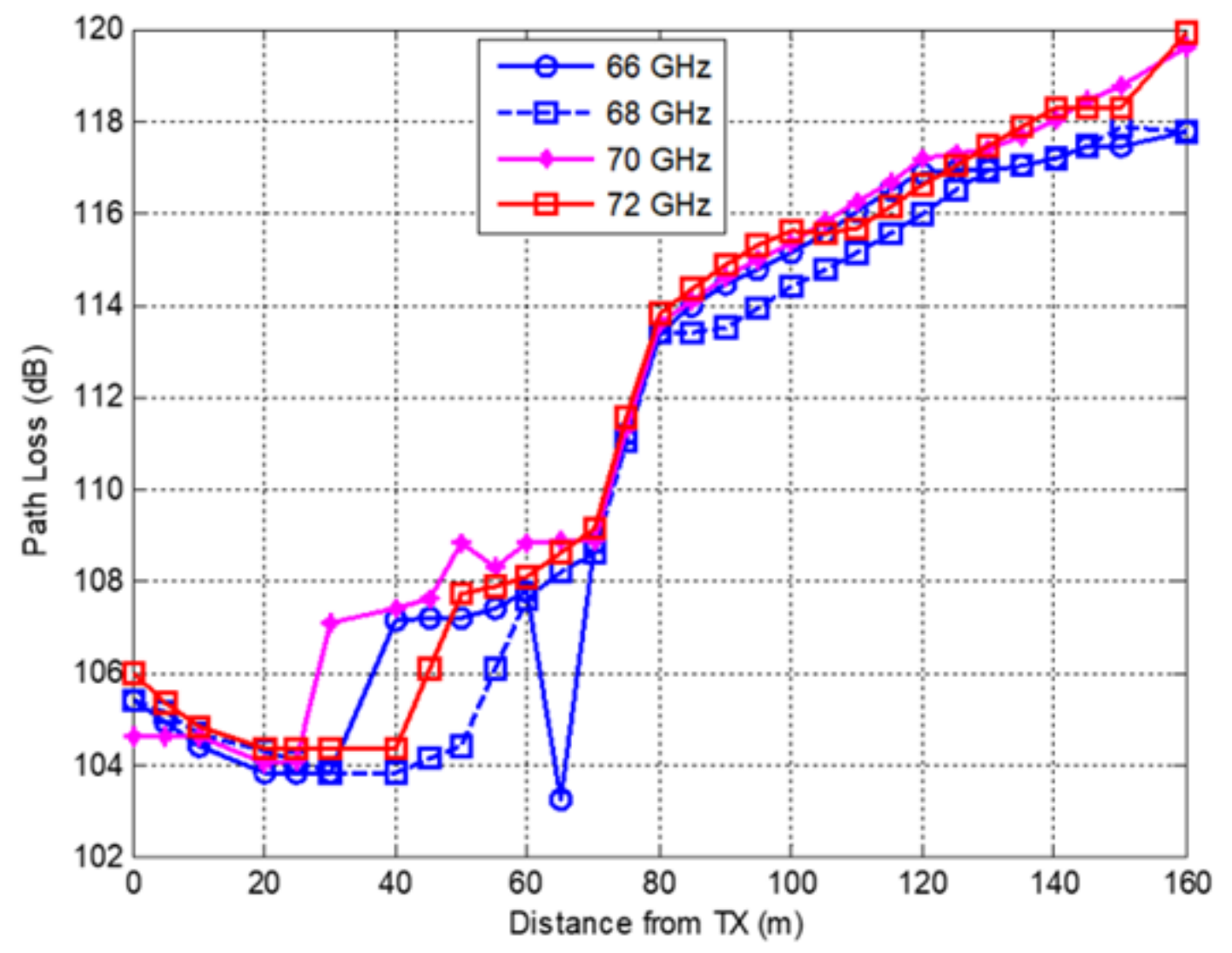

Figure 4

Path loss from TX1 site 1 to RX2 at different frequency 


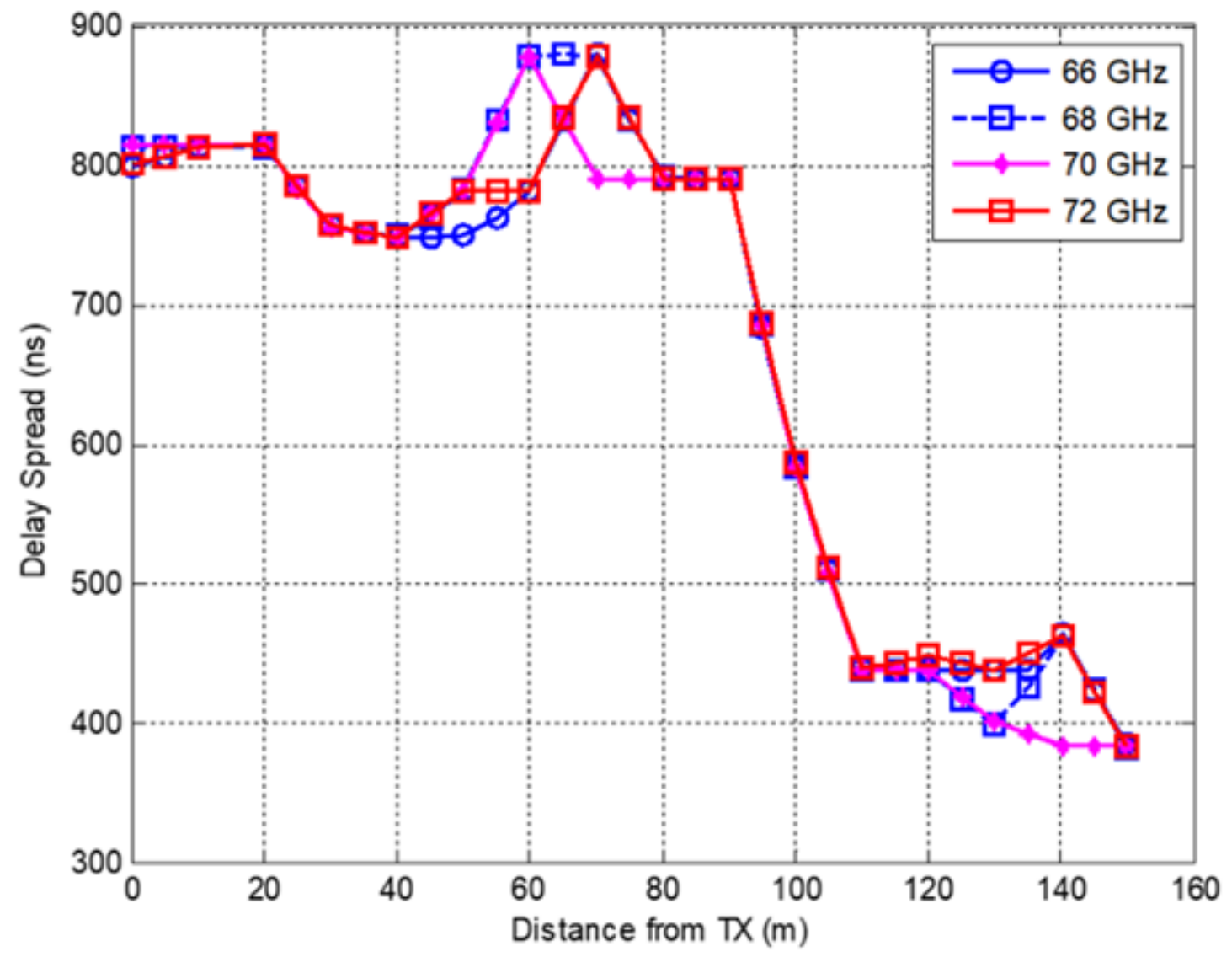

Figure 5

Delay Spread versus distance at different frequency

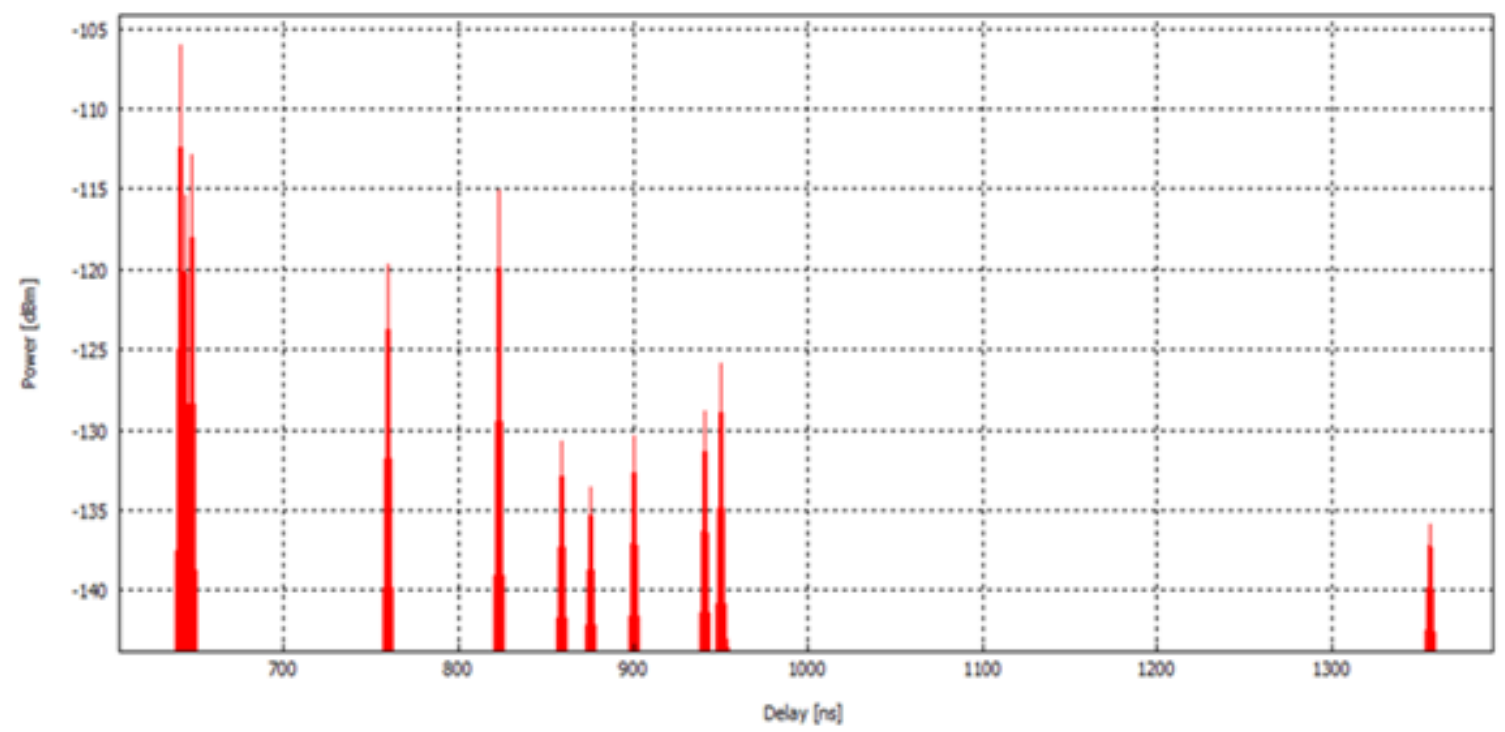

Figure 6 
Power delay profile at $\mathrm{T} \times 1-\mathrm{R} \times 2$ separation 